\title{
Perisynaptic Schwann Cells of the Vertebrate Motor Endplate Bear Modified Cilia
}

\author{
TILMAN VOIGT, ${ }^{1} *$ WOLFGANG DAUBER, ${ }^{2}$ AND ULRIKE KOHLER ${ }^{2}$ \\ ${ }^{1}$ Institute of Anatomy, University of Fribourg, 1700 Fribourg, Switzerland \\ ${ }^{2}$ Institute of Anatomy, Eberhard-Karls-University Tübingen 72074 Tübingen, Germany
}

KEYWORDS perisynaptic Schwann cell; modified cilia; Golgi apparatus; motor endplate

\begin{abstract}
Perisynaptic Schwann cells (PSCs), descendants of the myelinating Schwann cells, cover the axon terminal of the vertebrate motor endplate of the skeletal muscle fiber. PSCs are assumed to support the function of the axon terminal. This function suggests a net material transport in the direction of the axon terminal. Morphologically it is to be expected that these cells have a cytoskeleton aligned to the axon terminal. Investigations clarifying this statement have not yet been undertaken. From previous investigations we know, however, that the PSCs have a microtubule-organizing center, which is a part of this cytoskeleton. The centrioles of the organizing center may also participate in the formation of a modified cilium structure whose function is unknown. In the present investigation, characteristic ultrastructural features of the modified cilium structure and its relationship to the Golgi apparatus and the axon terminal are presented. A function for the modified cilium structure is discussed.
\end{abstract}

\section{INTRODUCTION}

In vertebrates, perisynaptic Schwann cells (PSCs) are involved in the building of the motor endplate (MEP) of skeletal muscle fibers (for review, see Salpeter 1987b). Because PSCs express Schwann cell-specific proteins, it is assumed that they are related to myelinating Schwann cells (Georgiou and Charlton, 1999). The PSCs cover over the axon terminal without myelinization and are thought to support the functions of the axon terminal (for review, see Salpeter, 1987b). This would require a directed net transport in the direction of the axon terminal. According to that, it can be shown by light-microscope that Schwann cell-specific membrane proteins are preferentially incorporated into the Schwann cell membrane facing the axon terminal (Georgiou and Charlton, 1999). From lightmicroscopic investigations at myelinating Schwann cells it is known that in these cells the transport occurs bilaterally to the nodes of Ranvier (Kidd et al., 1994). In order to understand the directed transport processes, the orientation of the cytoskeleton and the organization of the organelles is important. From other cells it is known that microtubules participate in this function (Cole and Lippincott Schwartz, 1995). In many cell types microtubules are nucleated from a microtubule-organizing center that forms a network throughout the cytoplasm (for review, see Cole and Lippincott Schwartz, 1995). Nucleation at a microtubule-organizing center has not yet been verified by light microscopy for myelinating Schwann cells (Kidd et al., 1994). Nonetheless, a modified cilium structure which is a presumed part of the cytoskeleton could be found in connection with the centriole in myelinating Schwann cells (Morris et al., 1972). In our ultrastructural investigations of MEPs of mammals (Dauber et al., 2000) and frogs (Voigt et al., 2000), we have occasionally observed cilium-like structures in the PSCs.
With further evaluation of serial sections we show for the first time a modified cilium structure. The characteristic features of this cilium-like structure in Schwann cells of the MEP of skeletal muscle fibers of mice and different frog species are morphologically described.

\section{MATERIALS AND METHODS}

The investigations were carried out using the diaphragm dissected from mice and the sartorius muscle dissected from frogs (Dauber and Meister, 1986; Dauber et al., 1999, 2000; Voigt et al., 2002). Unless otherwise mentioned, a 0.1 mol cacodylate buffer was employed as buffer solution. Anesthetized mice $(\mathrm{n}=7$; NMRI + wildtype) were perfused and fixed with $2.5 \%$ glutaraldehyde from the left ventricle (for further details, see Dauber and Meister, 1986). The middle third of the diaphragm, including the MEPs (Bowden and Duchen, 1976), were divided into portions and postfixed at $4^{\circ} \mathrm{C}$ by one of the following procedures: 1) our routine procedure using $1 \%$ osmium tetroxide for 2 hours (Dauber and Meister, 1986); 2) the imidazoleosmium procedure using $1 \%$ osmium tetroxide in 0.1 mol imidazole for 1 hour (Voigt et al., 2002); and (3) lanthanum incubation using $1 \%$ osmium tetroxide with $0.08 \%$ potassium ferrocyanide for 3 hours and incubation thereafter for 1.5 hours in $1 \%$ lanthanum nitrate (Dauber et al., 2000).

The sartorius muscles of the frogs (Xenopus laevis $\mathrm{n}=1$, Rana esculenta $\mathrm{n}=1$, and Rana temporaria $\mathrm{n}=$ 3) were fixed with $2.5 \%$ glutaraldehyde and postfixed

*Correspondence to: Dr. Tilman Voigt, Institute of Anatomy, Rte AlbertGockel 1, CH-1700 Fribourg, Switzerland. E-mail: tilman.voigt-gawatz@unifr.ch Received 11 June 2003; accepted in revised form 1 November 2003 DOI 10.1002/jemt.20023 


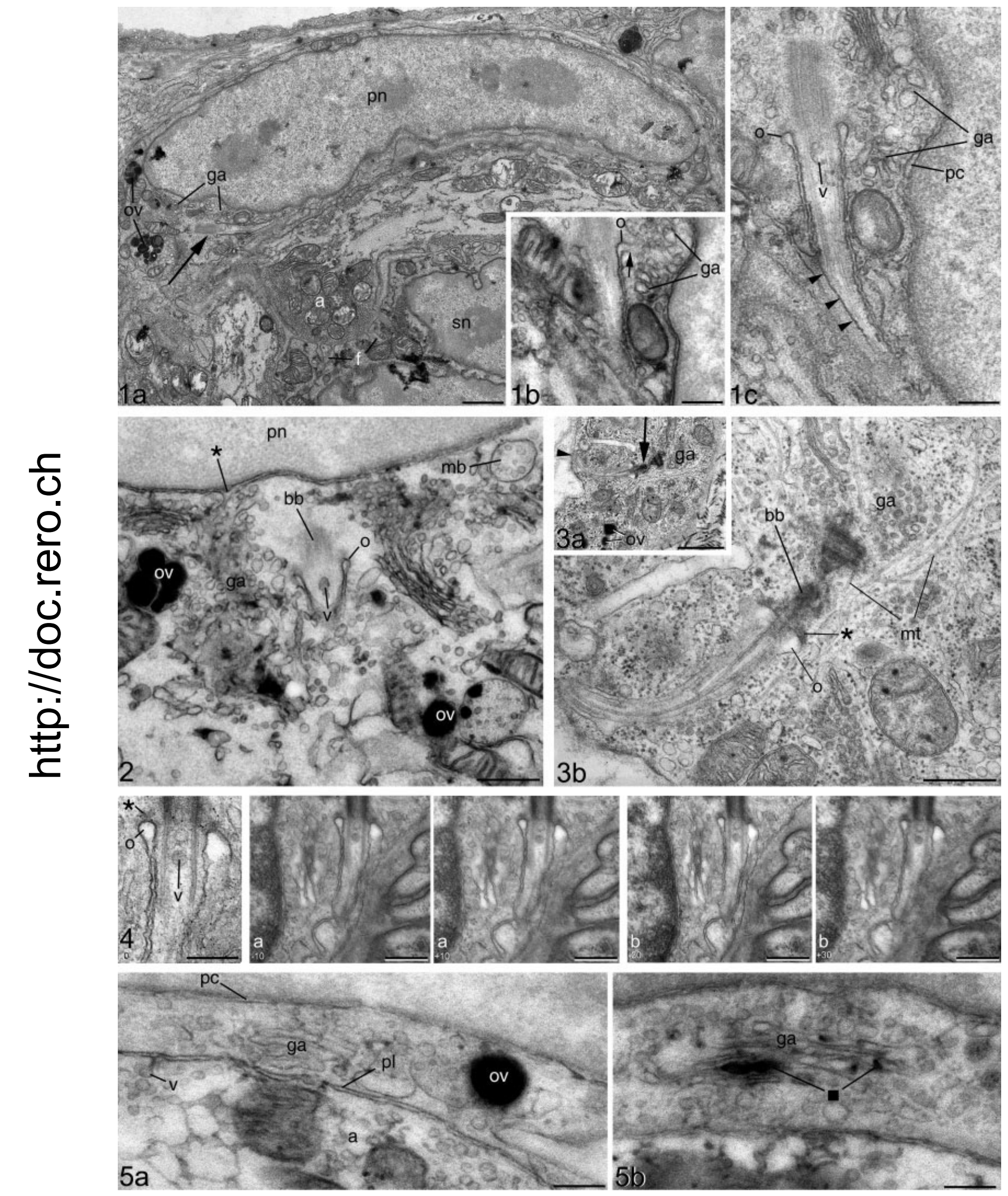

Figs. 1-5. 
for 3 hours at $4^{\circ} \mathrm{C}$ in $1 \%$ osmium tetroxide with $0.08 \%$ potassium ferrocyanide (for further details, see Voigt et al., 2000).

All samples were dehydrated and embedded in EPON. For locating MEPs in longitudinal and crosssections, two different search techniques were applied. Unlike in frogs, MEPs can be identified in semithin sections of longitudinal embedded muscle tissue of mammals by means of the accumulation of big rounded sole plate nuclei in areas densely populated by mitochondria (Dauber et al., 1999). A series of 25 thin $(100 \mathrm{~nm})$ sections were cut from theses areas. Both in longitudinal sections of frog muscles and in cross sections of mouse and frog muscles, sole plate nuclei and muscle fiber nuclei cannot be distinguished due to their shape. Thus, in these cases myelinated axons between muscle fibers are examined in semithin sections and in each case a series of 50 thin $(100 \mathrm{~nm})$ sections were cut. In order to overcome the problem of grid bars obscuring the areas of interest, the sections were mounted on slotted grids. The film stability was increased by the addition of $25 \mu \mathrm{l}$ dibutyl phthalate to $50 \mathrm{ml} 0.5 \%$ pioloform (Kneissler, 1997).

The sections of muscle tissue fixed according procedure 1 were stained with uranyl acetate and lead citrate (Reynolds, 1963); sections of muscle tissue fixed according to procedures 2 and 3 were analyzed in the unstained state, i.e., without further section staining. The sections were analyzed with a LEO912 Omega electron microscope.

\section{RESULTS}

The Golgi apparatus of PSCs surrounds the centrioles in proximity to the cell nucleus (Figs. 1,2). One of the centrioles forms the basal body of a structure embedded in the cytoplasm close to the cell nucleus. The cilium-like structure is surrounded by the Golgi apparatus, measuring $\sim 1.25 \mu \mathrm{m}$ in length and $\sim 240$ $\mathrm{nm}$ in diameter (Figs. 1, 3). Microtubules within this modified cilium surround its long axis in a coneshaped configuration. Along the long axis a couple of oval to round vesicles are centrally and sequentially located (Figs. 1c, 2, 4). The modified cilium structure forms a bent saber scabbard shape and its closed end is directed toward the cell surface without breaking it in any of our observations (Figs. 1c, 3a). Because of these characteristics, as well as the two missing central microtubules, we refer to these structures as modified cilium. These cilium-like structures are present within the PSCs of all examined skeletal muscle samples. Longitudinal as well as cross sections through the modified cilium of the PSCs appear in longitudinal (Figs. 1, 8) and cross-sections of the muscle (not shown). With every modified cilium the plasmalemma of the PSC is deeply invaginated and reaches up to the basal body where the invaginated membrane forms a torus-like ring opening, whose cross-sectional view has a torus thickness of $62-150$ $\mathrm{nm}$ (Figs. 1b,c, 2, 3b, 4). Both in the mammal and the frog, a diffuse felt-like staining is located on the intracellular surface of the torus-like ring opening (Figs. 3b, 4). Some section planes give the impression that this torus-like structure has small openings in the direction of the neighboring Golgi apparatus (Fig. 1b). We believe that these openings are not artifacts because the imidazole-osmium postfixation outlines fine membrane continuities especially well. The position of the Golgi apparatus around the centrioles also brings it proximal to the perinuclear cistern (Fig. 2). The membranes of the PSC facing the axon terminal run without basal membrane at intervals of $\sim 20 \mathrm{~nm}$ in parallel to one another (Figs. 5-8). In the mammalian PSC the large and numerous vesicles of the Golgi apparatus face the numerous vesicles of the axon terminal (Figs. 5a,b, 7). From both cell membranes, vesicles have been seen opening into the extracellular space between the facing membranes of the PSC and nerve cell (Fig. 6a,b). After imidazole-osmium postfixation, both osmiophilic vacuoles in proximity to the Golgi apparatus (Figs. 1, $2,3 a)$ and osmiophilic precipitates in the middle of the Golgi lamellae can be found (Fig. 5b). No osmio-
Fig. 1. a: Section of a mouse MEP. The body of the PSC caps the axon terminal (a). A modified cilium $(\rightarrow$ ) is situated proximal to the nucleus (pn) and Golgi apparatus (ga) of the PSC; osmiophilic vacuole (ov); sole plate nucleus (sn); subsynaptic folds (f). b: subsequent section of a with a $35^{\circ}$ tilt. The torus-like plasmalemmal widening (o) of the modified cilium opens to $(\rightarrow)$ the Golgi apparatus (ga). c: Detail of a. The modified cilium is surrounded by the Golgi apparatus (ga) which lies proximal to the perinuclear cistern (pc). The invaginated cell membrane surrounds the cilium shaft and forms a torus-like opening (o) at the base of the modified cilium. Vesicles (v) are situated in the center of the modified cilium instead of the two central microtubules found in normal cilia. The modified cilium does not protrude beyond the surface of the cell body $(\boldsymbol{\nabla})$. Imidazole-osmium procedure, section not stained. Scale bars $=1 \mu \mathrm{m}(\mathbf{a}), 250 \mathrm{~nm}(\mathbf{b}), 250 \mathrm{~nm}(\mathbf{c})$.

Fig. 2. Structure within a PSC of a mouse MEP. The perinuclear cistern (*) opens directly to the Golgi apparatus (ga) that surrounds the modified cilium; basal body (bb), multivesicular body (mb), osmiophilic vacuole (ov), torus-like opening (o) at the base of the cilium shaft, vesicle lying centrally (rather than the microtubules) (v). Imidazole-osmium procedure, section not stained. Scale bar $=500 \mathrm{~nm}$.

Fig. 3. a: Section of a PSC of an MEP of Xenopus laevis. The modified cilium $(\rightarrow)$ does not extend beyond the surface of the PSC; end of the modified cilium extending to the cell surface ( $)$; Golgi apparatus (ga); osmiophilic vacuole (ov). b: Detail from a. One of the two centrioles forms the basal body (bb) of the modified cilium; toruslike opening (o); felt-like staining (*). Microtubules (mt) radiate from the basal body partially in the direction of the Golgi apparatus (ga). Osmium potassium ferrocyanide postfixation, section stained. Scale bars $=1 \mu \mathrm{m}(\mathbf{a}), 500 \mathrm{~nm}(\mathbf{b})$.

Fig. 4. a,b: Base of a modified cilium of a PSC in a mouse MEP Vesicles (v) are situated in the center of the modified cilium instead of the two central microtubules. At the open base of the modified cilium the invaginated cell membrane forms a torus-like opening (o) that is stained with a diffuse felt-like appearance (*). Routine procedure, section stained, tilt angle $0^{\circ}$; bar $=250 \mathrm{~nm}$. a,b: After section tilting the central vesicles remain between the microtubules. Corresponding figures were placed adjacent to each other; tilt angle $(\mathbf{a}) \pm 10^{\circ} ;(\mathbf{b})$ $\pm 30^{\circ} ;$ scale bar $=500 \mathrm{~nm}$.

Fig. 5. a,b: Subsequent sections through the PSC of a mouse MEP. The Golgi apparatus (ga) of the PSC is directly situated between the nuclear envelope and the plasmalemma of the PSC (pl). The gap between the plasmalemma of the PSC and the axon terminal (a) has similar gap dimensions as the gap between the inner and external membranes of the perinuclear cistern (pc); osmiophilic vacuole (ov). a: At the axolemma, a vesicle (v) opens into the gap between the PSC and the axon terminal. b: In some sections, the Golgi lamellae show osmiophilic inclusions (a). Imidazole-osmium procedure, section not stained. Scale bars $=200 \mathrm{~nm}$ 


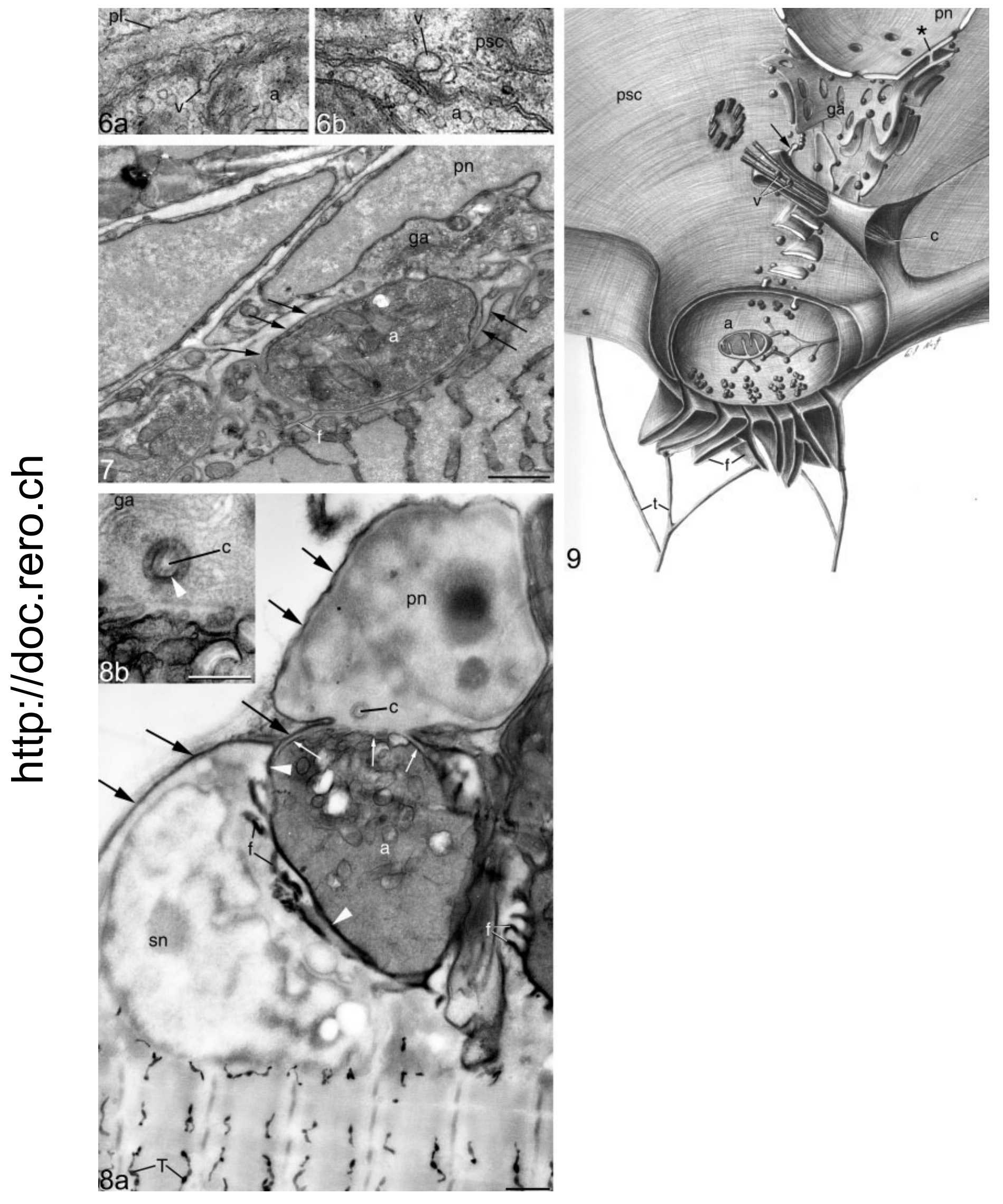

Figs. 6-9. 
philic precipitates, however, were found in this extracellular space. Even after successful lanthanum incubation this extracellular space between the PSC and the axon terminal is not filled with lanthanum (Fig. 8a,b). The results, found in both mouse and frog, are summarized graphically in Fig. 9.

\section{DISCUSSION}

The present investigation has shown that perisynaptic Schwann cells (PSCs) contain modified cilia. They are different from "normal" cilia because they are missing the two central microtubules normally paralleling its long axis. In their place, a pair of vesicles are sequentially situated on the long axis. The centriole areas in proximity to the nuclei appear to be the origin of these modified cilia. In all PSCs examined in every healthy animal, modified cilia were found. We assume that these modified cilia are a normal cell structure in these PSCs and their existence is not a degenerative modification occurring in the area of the MEP (Dennis and Miledi, 1974; Glazer et al., 1978).

Comparable structures were observed in glia cells outside of the ependyma in the central and peripheral nervous system and they were designated "cilia" (Grillo and Palay, 1963; for review, see Peters et al., 1991). Their organization, dimensions, and integration into the cell environment as described previously agree with our findings (Grillo and Palay, 1963).

The existence of one only $2-3-\mu \mathrm{m}$ long cilium was interpreted as the transition of the cell into the $G_{0}$ stage in investigations of cell cultures (3T3, rat spleen) (Tucker et al., 1979). Thus, the existence of the modified cilia in PSCs of adult mice and frogs can be interpreted as a sign of a very small division rate. In PSC, no cell divisions were observed during the contact between neurons and Schwann cells (for review, see Reynolds and Woolf, 1993), nor did cell division occur in response to denervation (Connor et al., 1987). The number of individually observed PSCs in adult mice in vivo remains unchanged over weeks and also after denervation (O'Malley et al., 1999). On the other hand, the number of PSCs is assumed to increase in young muscles both through migration and through mitosis (Love and Thompson, 1998).

According to the cell type, the position and size of the Golgi apparatus can vary. The Golgi apparatus is assumed to be distributed along the microtubules bundles in myelinating Schwann cells (Kidd et al., 1994). These microtubule bundles are assumed to lie perinuclear and not to converge in the direction of the nucleus and/or the centrioles (Kidd et al., 1994). In the PSCs as well as in many polarized eukaryotic cells (Thyberg and Moskalewski, 1999) the Golgi apparatus is juxtanuclear and is arranged around the centrioles. The presumption that the development of an extensive Golgi apparatus in PSC signals regeneration (Salpeter, 1987a) is contradicted by our observation of the limited Golgi apparatus localization proximal to the axon terminal in healthy animal specimens. Even if there is no evidence for a vesiclelike transmitter release from glia cells (Martin, 1992), the Golgi apparatus we have described in the PSC suggests a material transport in the direction of the plasmalemma facing the axon terminal (Cole and Lippincott Schwartz, 1995; Drubin and Nelson, 1996; Rogalski and Singer, 1984). Indeed the distance between the PSC and the axon has a similar dimension as a chemical synapse (Gray, 1995). However, we could not verify characteristics of chemical synapse such as the presence of membrane thickening on the axonal side. Also, the few vesicles in contact with the plasmalemma facing the axon terminal cannot be interpreted as a characteristic of a chemical synapse. Nonetheless, the incorporation of myelin-associated glycoprotein in the cell membrane at the contact zone with the axon terminal was demonstrated, even though PSC do not form any myelin sheets (Georgiou and Charlton, 1999). This glycoprotein is assumed to be involved in a dynamic mechanical link between the cytoskeleton of the PSC and the axon terminal (Kursula et al., 2001). Unlike in myelinating Schwann cells, an alignment of the described cell organelles in the direction of the axon terminal exists in PSC so that by means of the cytoskeleton a directed dynamic mechanical link could be in operation. The open-ended base of the described modified cilium is considered to be a microtubule organizing center. Currently, no satisfactory explanation of the function of these modified cilia in PSCs has been published.
Fig. 6. a,b: Cell structures seen in the PSC of the MEP of a mouse skeletal muscle fiber. a: At the axolemma a vesicle (v) opens into the gap between the PSC and the axon terminal (a); plasmalemma of the PSC (pl). b: On sides of the PSC (psc) a vesicle (v) opens to the gap between the PSC and the axon terminal (a). Routine procedure, section stained. Scale bars $=200 \mathrm{~nm}$.

Fig. 7. Fine structures of the PSC of an MEP in a cross section through a mouse skeletal muscle fiber. The cell body of the PSC caps the axon terminal (a), nucleus of the PSC (pn). In part the axon terminal is only covered by a cytoplasmic sheet of the PSC $(\rightarrow)$. The Golgi apparatus (ga) is proximal to the nucleus of the PSC and the axon terminal; subsynaptic fold (f). Imidazole-osmium procedure, section not stained. Scale bar $=1 \mu \mathrm{m}$.

Fig. 8. a: Overview of a MEP after lanthanum incubation. Through the lanthanum incubation, the extracellular space in the muscle fiber-T-system (t) and subsynaptic folds (f) as well as the primary synaptic gutter $(\boldsymbol{\nabla})$ are marked. Whereas in the basal membrane of the sole plate, the axon terminal (a) and the PSC (large arrows) are marked by lanthanum, the gap (small arrows) between the perisynaptic Schwann cell and axon terminal is unmarked; nucleus of the PSC (pn); sole plate nucleus (sn). The slightly diagonal sectional view through a cilium shaft (c) is situated below the nucleus of the PSC. Lanthanum incubation, section not stained. Scale bar = $1 \mu \mathrm{m}$. b: Detail of a. The slightly diagonal view of the cilium shaft (c) is surrounded by the Golgi apparatus (ga), membrane of the cilium ( $)$. Lanthanum incubation, section not stained. Scale bar $=500 \mathrm{~nm}$. Fig. 9. Three-dimensional graphic representation of an MEP with a PSC (psc) that caps the axon terminal (a); subsynaptic folds (f) with T-tubules $(t)$ of the sole-plate. For better visualization of the modified cilium in the PSC, only a section through the cell nucleus (pn) and through the Golgi apparatus (ga) with its relations to the modified cilium $(\rightarrow)$ and/or the perinuclear cistern $(*)$ are represented. The modified cilium (c) does not extend beyond the cell surface. A part of the invaginated cell membrane was removed in order to visualize the interior of the modified cilium with its microtubules and the vesicles sequentially lying along the central axis $(\mathrm{v})$. 


\section{REFERENCES}

Bowden RRM, Duchen LW. 1976. The anatomy and pathology of the neuromuscular junction. In: Zaimis E, Maclagan J, editors. Handbook of experimental pharmacology, vol. 42. Neuromuscular junction. Berlin: Springer. p 23-97.

Cole NB, Lippincott Schwartz J. 1995. Organization of organelles and membrane traffic by microtubules. Curr Opin Cell Biol 7:55-64.

Connor EA, McMahan UJ, Marshall RM. 1987. Cell accumulation in the junctional region of denervated muscle. J Cell Biol 104:109120.

Dauber W, Meister A. 1986. Ultrastructure of junctional folds of motor end plates in extensor digitorum longus muscles of mice. J Ultrastruct Mol Struct Res 97:158-164.

Dauber W, Voigt T, Heini A. 1999. Junctions between subsynaptic folds and rough sarcoplasmic reticulum of muscle fibres. J Muscle Res Cell Motil 20:697-701.

Dauber W, Voigt T, Härtel X, Meyer J. 2000. The t-tubular network and its triads in the sole plate sarcoplasm of the motor end-plate of mammals. J Muscle Res Cell Motil 21:443-449.

Dennis MJ, Miledi R. 1974. Electrically induced release of acetylcholine from denervated Schwann cells. J Phys 237:431-452.

Drubin DG, Nelson WJ. 1996. Origins of cell polarity. Cell 84:335344.

Georgiou J, Charlton MP. 1999. Non-myelin-forming perisynaptic Schwann cells express protein zero and myelin-associated glycoprotein. Glia 27:101-109.

Glazer EJ, Baker T, Riker-WF J. 1978. The neuropathology of DFP at cat soleus neuromuscular junction. J Neurocytol 7:741-758.

Gray H. 1995. Gray's anatomy, 38th ed. Williams PL, Bannister LH, Berry MM, Collins P, Dyson M, Dussek JE, Ferguson MWJ, editors. London: Churchill Livingstone.

Grillo MA, Palay SL. 1963. Ciliated Schwann cells in tha autonomic nervous system of the adult rat. J Cell Biol 16:430-436.

Kidd GJ, Andrews SB, Trapp BD. 1994. Organization of microtubules in myelinating Schwann cells. J Neurocytol 23:801-810.

Kneissler U. 1997. Transmissions-Elektronenmikroskopie Teil2: Pioloform BM 18 zur Optimierung der physikochemischen Eigenschaften von Trägerfolien. Die Fachzeitschrift für den Technischen Assitenten in der Medizin 12:322-324.

Kursula P, Lehto V-P, Heape AM. 2001. The small myelin-associated glycoprotein binds to tubulin and microtubules. Mol Brain Res 87:22-30.
Love FM, Thompson WJ. 1998. Schwann cells proliferate at rat neuromuscular junctions during development and regeneration. J Neurosci 18:9376-9385.

Martin DL. 1992. Synthesis and release of neuroactive substances by glia cells. Glia 5:81-94.

Morris JH, Hudson AR, Weddell G. 1972. A study of degeneration and regeneration in the divided rat sciatic nerve based on electron microscopy. II. The development of the "regenerating unit." Zeitschrift fuer Zellforschung 124:103-130.

O'Malley JP, Waran MT, Balice-Gordon RJ. 1999. In vivo observations of terminal Schwann cells at normal, denervated, and reinnervated mouse neuromuscular junctions. J Neurobiol 38:270-286.

Peters A, Palay SL, Webster HD. 1991. The fine structure of the nervous system. Neurons and their supporting cells. Oxford: Oxford University Press.

Reynolds ES. 1963. The use of lead citrat at high $\mathrm{pH}$ as an electronopaque stain in electron microscopy. J Cell Biol 17:208-212.

Reynolds ML, Woolf CJ. 1993. Reciprocal Schwann cell-axon interactions. Curr Opin Neurobiol 3:683-693.

Rogalski AA, Singer SJ. 1984. Associations of elements of the Golgi apparatus with microtubules. J Cell Biol 99:1092-1100.

Salpeter MM. 1987a. Development and neural control of the neuromuscular junction and of the junctional acetylcholin receptor. In: Salpeter MM, editor. Neurology and neurobiology, vol. 23. The vertebrate neuromuscular junction. New York: Alan R. Liss. p 55116.

Salpeter MM. 1987b. Vertebrate neuromuscular junction: general morphology, molecular organization, and functional consequences. In: Salpeter MM, editor. Neurology and neurobiology, vol. 23. The vertebrate neuromuscular junction. New York: Alan R. Liss. p 1-54.

Thyberg J, Moskalewski S. 1999. Role of microtubules in the organization of the Golgi complex. Exp Cell Res 246:263-279.

Tucker RW, Pardee AB, Fujiwara K. 1979. Centriole ciliation is related to quiescence and DNA synthesis in 3T3-cells. Cell 17:527535.

Voigt T, Dauber W, Härtel X, Schönemann R. 2000. On the connections between the T-system and the subsynaptic folds in the motor end plate of amphibians. J Neurocytol 30:157-164.

Voigt T, Dauber W, Bensemann-Ryvkin I, Härtel X. 2002. Increasing membrane contrast by means of imidazole-osmium post-fixation as exemplified by skeletal muscle fiber. Microsc Res Tech 58:121-124. 